\title{
Detection of extremely low concentration waterborne pathogen using a multiplexing self-referencing SERS microfluidic biosensor
}

\author{
Chao Wang ${ }^{1}$, Foram Madiyar ${ }^{2}$, Chenxu Yu ${ }^{1 *}$ (D) and Jun $\mathrm{Li}^{2}$
}

\begin{abstract}
Background: It is challenging to achieve ultrasensitive and selective detection of waterborne pathogens at extremely low levels (i.e., single cell/mL) using conventional methods. Even with molecular methods such as ELISA or PCR, multi-enrichment steps are needed which are labor and cost intensive. In this study, we incorporated nano-dielectrophoretic microfluidic device with Surface enhanced Raman scattering (SERS) technique to build a novel portable biosensor for easy detection and characterization of Escherichia coli 0157:H7 at high sensitivity level (single cell/mL).
\end{abstract}

Results: A multiplexing dual recognition SERS scheme was developed to achieve one-step target detection without the need to separate target-bound probes from unbound ones. With three different SERS-tagged molecular probes targeting different epitopes of the same pathogen being deployed simultaneously, detection of pathogen targets was achieved at single cell level with sub-species specificity that has not been reported before in single-step pathogen detection.

Conclusion: The self-referencing protocol implements with a Nano-dielectrophoretic microfluidic device potentially can become an easy-to-use, field-deployable spectroscopic sensor for onsite detection of pathogenic microorganisms.

\section{Background}

Pathogen detection and identification is of the utmost importance for medicine, food safety, public health and security, and water and environmental quality control [1]. The World Health Organization (WHO) identified that contaminated water serves as a mechanism to transmit communicable diseases such as diarrhea, cholera, dysentery, typhoid and guinea worm infection. Except for poor water, sanitation and hygiene services (WASH) conditions in communities and institutional settings, slow detection strategies have also been exacerbating the spread of those infectious diseases. Timing is extremely important in pathogen detection and the delay or inaccurate diagnosis of the pathogenic infection is always the primary cause of mortality or serious illness. Traditional and standard pathogen detection methods rely on

\footnotetext{
* Correspondence: chenxuyu@iastate.edu

'Agricultural and Biosystems Engineering Department, lowa State University, Ames, IA 50011, USA

Full list of author information is available at the end of the article
}

off-line laboratory procedures (consist of multiple cultural enrichment steps, isolation of bacterial colonies, identification) and may take up to 8 days to yield an answer [2]. This slow process clearly can't provide a sufficient protection from exposure to water borne pathogens within public drinking water. Outside traditional culturing, many methods have been developed to promote the detection efficacy, such as polymerase chain reaction (PCR), enzyme-linked immunosorbent assay (ELISA), and surface plasmon resonance (SPR) sensors [3-6]. These techniques provide high selectivity and reliability; however, they usually require intensive sample preparation and special equipment and trained users [7]. Furthermore, in reality, the competitor organisms in water samples can cross-react with detection systems, rendering false-positive results, or can grow to levels that will mask target organisms. Hence, there is a compelling need for the development of easy-to-use biosensors that could give highly sensitive and reliable 
detection results, and even allow on-site field monitoring [8].

Surface-enhanced Raman scattering (SERS), as a labelfree/non-destructive optical technique, has been widely used in pathogen discrimination [9-12]. The distinct "fingerprinting" Raman spectra of microorganisms can be enhanced at rough noble metal nanostructures' surfaces, which is essentially important in pathogen detection since discrimination of different bacterial species and strains is difficult. Recently, various nanostructures with different surface features have been employed to amplify the enhancement of SERS signals in bacterial identifications at cellular and molecular levels. However, it is still a challenge to obtain repeatable and reproducible SERS spectroscopic results at complicated experimental conditions. The degree of metallic nanoparticles aggregation, the different size of metal colloids, and the inhomogeneous distributions of nanoparticles on cells all affect the SERS signal reproducibility. To overcome those limitations, specific antibodies and Raman tags molecules are introduced into nanostructures to probe the target biomolecules and produce a high-specific and reproducible SERS signals [13-15]. However, the simultaneous presence of nanoparticles, SERS reporters, and biological samples generates highly overlapping and complex spectra which make it difficult to identify the target bacteria. Therefore, it is necessary to integrate statistical analysis techniques into bacterial SERS discrimination for data mining [14, 16-20].
Herein, we developed the concept of self-referencing mechanism that utilized SERS molecular probes to achieve target bacteria detection in one single step with high reliability brought by a novel multiplex targeting scheme, and integrated multivariate statistical analysis methods to simplify the superimposed SERS spectra for rapid and accurate diagnostics of water samples. To further improve the limit of detection (LOD) in the pathogenic bacteria detection strategy, and to facilitate possible deployment as on-site detection apparatus, a bacterial concentration mechanism based on nanodielectrophoretic (Nano-DEP) enrichment was integrated with the SERS signal acquisition/analysis to yield a microfluidic sample preparation platform (Fig. 1). Although in recent years, quite a few reports on DEPbased microfluidic biosensors have been published [2124], including a few with SERS as the detection mechanism [25-28], almost all of the relevant past work used microbial samples with high concentrations $\left(>10^{6} \mathrm{CFU} /\right.$ $\mathrm{mL}$ ) for DEP operations. In this study, we investigated samples with microbial concentration at $1-10 \mathrm{CFU} / \mathrm{mL}$, which is more relevant in terms of potential practical applications, such as monitoring pathogens in drinking water.

\section{Methods}

\section{Chemical and biological materials}

Hexadecyltrimethylammoniumbromide (CTAB, $299 \%$ ); Gold(III) chloride trihydrate $\left(\mathrm{HAuCl}_{4} \cdot 3 \mathrm{H}_{2} \mathrm{O}, 99.9+\%\right)$;

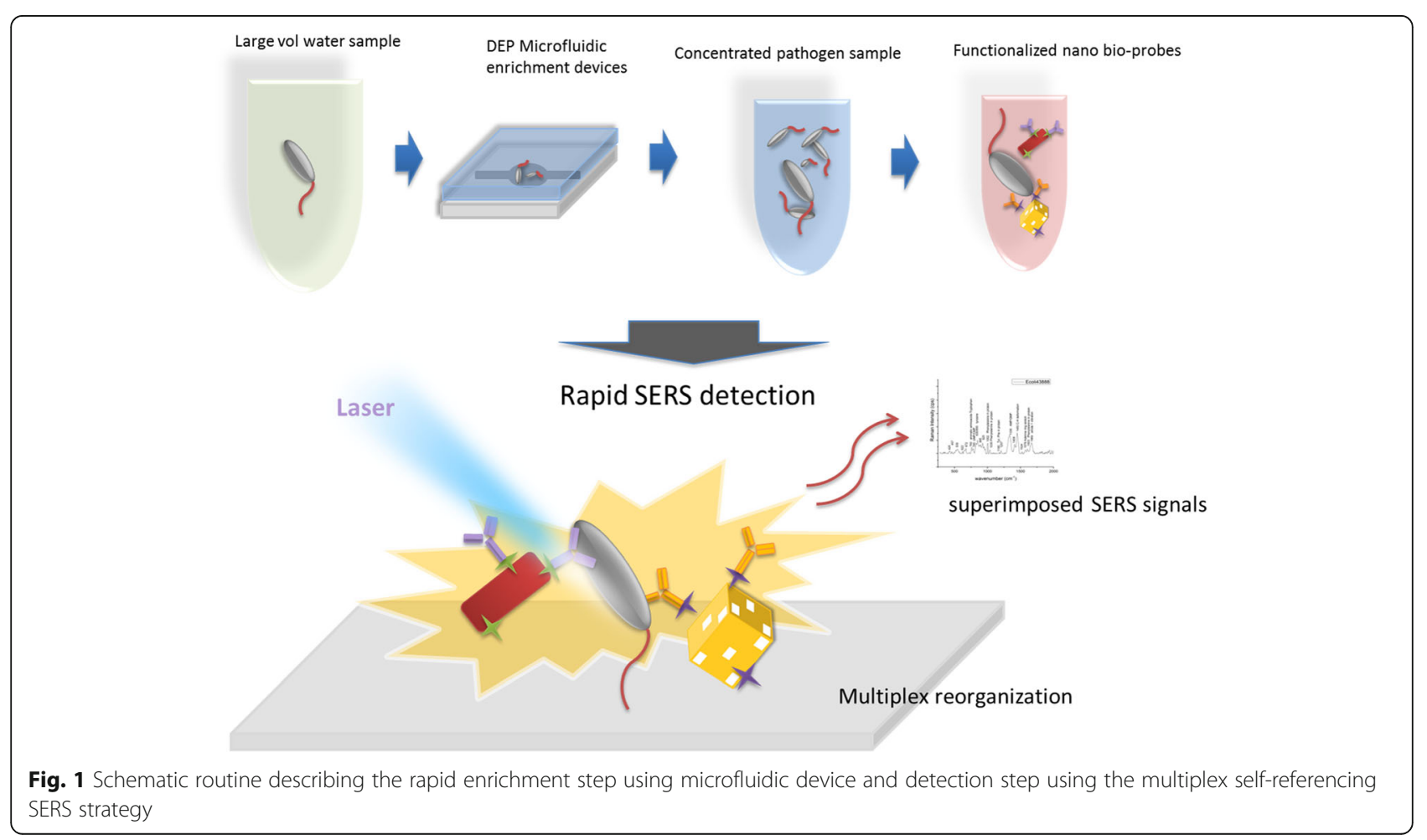


Sodium borohydride $\left(\mathrm{NaBH}_{4}, \geq 99 \%\right)$; Silver nitrate $\left(\mathrm{AgNO}_{3}, \geq 99 \%\right)$; L-Ascorbic acid (AA, $\left.\geq 99.0 \%\right)$; 4Aminothiophenol (4-ATP, 97\%); 3-Amino-1,2,4-triazole5-thiol (ATT, 95\%); Phosphate-buffered saline (PBS), 10× concentrate. Ethylene glycol (EG, 99\%), sodium sulfide $\left(\mathrm{Na}_{2} \mathrm{~S}, 99 \%\right)$; Polyvinylpyrrolidone (PVP, 99\%); 3Mercaptopropionic acid ( $\geq 99 \%)$. All reagents are purchased from Sigma-Aldrich. E.coli O157: H7 (No. 43888) and E.coli K12 (29425) frozen-dried strains were purchased from ATCC (Manassas, VA, USA). Anti-E.coli antibodies were purchased from Abcam (Cambridge, MA, USA). 18.2 M $\Omega . c m$ E-pure water is used for all regents' preparation.

\section{Bacterial sample preparation for Raman spectroscopic analysis}

Different bacterial strains were cultured in petri dishes of $60 \mathrm{~mm} \times 15 \mathrm{~mm}$ that have a layer of agar-based Luria Broth medium. After $18 \mathrm{~h} 37^{\circ} \mathrm{C}$ incubation, the desired bacteria colonies were inoculated in liquid Luria Broth medium for liquid culture. After $18 \mathrm{~h}$ incubation at $37^{\circ} \mathrm{C}$, bacterial solution was transferred to $15 \mathrm{~mL}$ centrifuge tubes and concentrated under 3000 RPM speed for $3 \mathrm{~min}$. After removing the supernatant, the dense pellets of bacteria were obtained for subsequent Raman identification tests or series dilution.

\section{Functionalization Gold nanorods (GNRs) with 4-ATP and ATT and antibodies}

GNRs were synthesized following the standard protocol in literatures [29]. $3 \mathrm{~mL}$ of $10 \mathrm{mM}$ 4-ATP and ATT $(\mathrm{pH}=2)$ was added into $24 \mathrm{~mL}$ GNR-CTAB with LSPR OD (optical density) $=6$. The mixture solution was kept in disposable scintillation vials at $60^{\circ} \mathrm{C}$ oil bath with 180 rpm stirring speed for around $19 \mathrm{~h}$. Then, functionalized GNRs solution was washed twice by centrifugation (6000 $\times$ g for $10 \mathrm{~min}$ ) with $20 \mathrm{mM}$ CTAB and $\mathrm{pH}=4$ pure water. Finally suspend the products in $0.25 \mathrm{~mL}$ water. For antibody conjugation, $0.75 \mu \mathrm{g}$ anti-E.coli O157:H7 mouse monoclonal antibodies (P3C6, ab75244) were incubated with $500 \mu \mathrm{L}$ GNR-4ATP (OD=11.2); $0.75 \mu \mathrm{g}$ another anti-E.coli O157:H7 mouse monoclonal antibodies (3011, ab20976) were incubated with $500 \mu \mathrm{L}$ GNR-ATT.

\section{Functionalization of Cage with 3-MPA and antibodies}

Gold cages were synthesized following the standard protocol in literatures [30]. The 3-MPA-gold nanocages were prepared by ligand-exchange reaction between 3MPA and PVP stabilized gold nanocages. The cages solution were diluted to $100 \mathrm{~mL}$ with $\mathrm{OD}=1.0$. Ligandexchange reaction was performed at room temperature by mixing the prepared cage solution with a $100 \mu \mathrm{L}$ of aqueous solution of $20 \mathrm{mM}$ 3-MPA under shaking treatment. The mixed solution was treated overnight under the room temperature. After centrifuging, the supernatant were removed. The pellet was washed with pure water for 1 time. For antibody conjugation, $0.75 \mu \mathrm{g}$ antiE.coli O157:H7 rabbit polyclonal antibodies (HRP ab68450 from Abcam) were incubated with $500 \mu \mathrm{L}$ Cage-3MPA.

\section{SERS measurement}

DXR Raman microscope (Thermo Scientific, Waltham, MA, USA) was used for Raman spectra acquisition with $780 \mathrm{~nm}$ excitation at $10 \mathrm{~mW}, 10 \times$ objective, and $25 \mu \mathrm{m}$ slit. The laser exposure time was $5 \mathrm{~s}$ and spectral resolution was $2.4-4.4 \mathrm{~cm}^{-1}$. Different batches of nanoprobes were used for each mixed sample to test the reproducibility of the SERS measurement. Several droplets of sample solutions were placed on gold-coated microscope slide, and multiple SERS spectra were obtained from different positions on each droplet. The OMNIC $^{\mathrm{Tm}}$ suite (Thermo Scientific, Waltham, MA, USA) was used for data processing. The focusing point in the colloidal state liquid sample is randomly selected for all collection in order to obtain a big and random database to fulfill the requirement in the following statistical analysis.

\section{Nano-DEP microfluidic device operation}

By using the microfluidic device, cell enrichment could be achieved in a continuous sample preparation step. Two different E.coli strains were mixed: E.coli O157:H7 (pathogenic) and E.coli K12 (non-pathogenic) and used to test the efficiency of the microfluidic device. The mixture was used so that the specificity of the selfreferencing mechanism in the following SERS measurement can be investigated. Two E. coli strains were diluted to extremely low concentrations and mixed together uniformly. $1 \mathrm{~mL}$ of the mixed cell suspension at $10^{\circ} \mathrm{CFU} / \mathrm{mL}$ was passed through the microfluidic device. At a flow rate of $1 \mu \mathrm{L}$ per min, it took about $17 \mathrm{~h}$ for the $1 \mathrm{~mL}$ sample to be processed. The concentrated samples then were collected.

\section{Statistical analysis}

The spectra were firstly baseline-corrected, smoothed and area normalized. An iterative polynomial background removal algorithm was implemented to remove background fluorescence from the Raman spectral data [31].

Principal components analysis (PCA) is a common statistical technique that is used to reduce the number of dimensions of data with a minimum loss of information [19]. The goal of PCA is to determine the data patterns and underlying factors that cause the similarities and differences of the original data without any prior knowledge. Those factors are orthogonal basis and called 
principal components (PCs). For each PC score, the influence (weight) of the original spectral data is found in its corresponding loading profile. In this study, PCA was performed using MatLab (Mathworks, Inc., Natick, MA).

\section{Result and discussion}

Multiple bioconjugated gold nanoparticles (AuNPs) as SERS nanoprobes for bacterial identification at $10 \mathrm{CFU} / \mathrm{mL}$

The mechanism of the self-referencing scheme of pathogen detection using two probes targeting two epitopes of the same pathogen is shown in Fig. 2. Only specific binding of nanoprobes to targets will yield detectable dual SERS signal (i.e., probe+target signals), non-specific binding or no-binding will not yield dual signals in this scheme, because only the specially designed SERS probes, made from functionalized anisotropic nanoparticles, can generate enough enhancement to the bacterialoriginated signal to make it SERS-detectable [23]. However, in the single epitope mechanism we reported earlier [32], the chance of probes not binding properly to the targets was still quite high. To further improve the detection specificity and signal enhancement by reducing the miss-binding, we developed a three-epitope detection scheme to avoid the antibody-antigen binding failures in one-epitope setup. Briefly, three different types SERS probes with monoclonal antibodies binding to three different epitopes on the same pathogen cell were deployed against a pathogen target, each of the probe-target binding events become more distinctive and specific due to the appearance of different SERS tag signals with various enhanced signature peaks. Three different Raman tag molecules (4-Aminothiophenol, 4-ATP; 3-Amino-1,2,4-triazole-5-thiol, ATT; and 3Mercaptopropionic acid, 3-MPA) are selected as probe reporters for nanoparticle functionalization, because of their large Raman cross section, and favorable chemistry for antibody conjugation. The three selected Raman tag molecules have weak signals in the microbial spectral range so that the microbial signals would not be overwhelmed by the enhanced Raman tags peaks in SERS spectral analysis.

Two anisotropic AuNPs, nanorods and nanocages, were employed as SERS enhancers because anisotropic particles show stronger electromagnetic enhancement compared to isotropic structures [33]. After covalently coupling the three tags molecules on nanostructures, respectively (4-ATP and ATT conjugated to nanorods, and 3-MPA conjugated on nanocages), three different antiE.coli O157: $\mathrm{H} 7$ antibodies were conjugated to the tags molecules by diazo bonding reaction and EDC/NHS bonding reaction. The SERS spectra of three nanoprobes are shown in Fig. 3. By comparing the Raman intensities of these three probes, the enhancement factors from diazo bonding conjugation (4-ATP-antibody and ATTantibody) were larger than EDC/NHS bonding (3-MPAantibody). The nanorod-4ATP-antibody showing the highest enhancement factor.

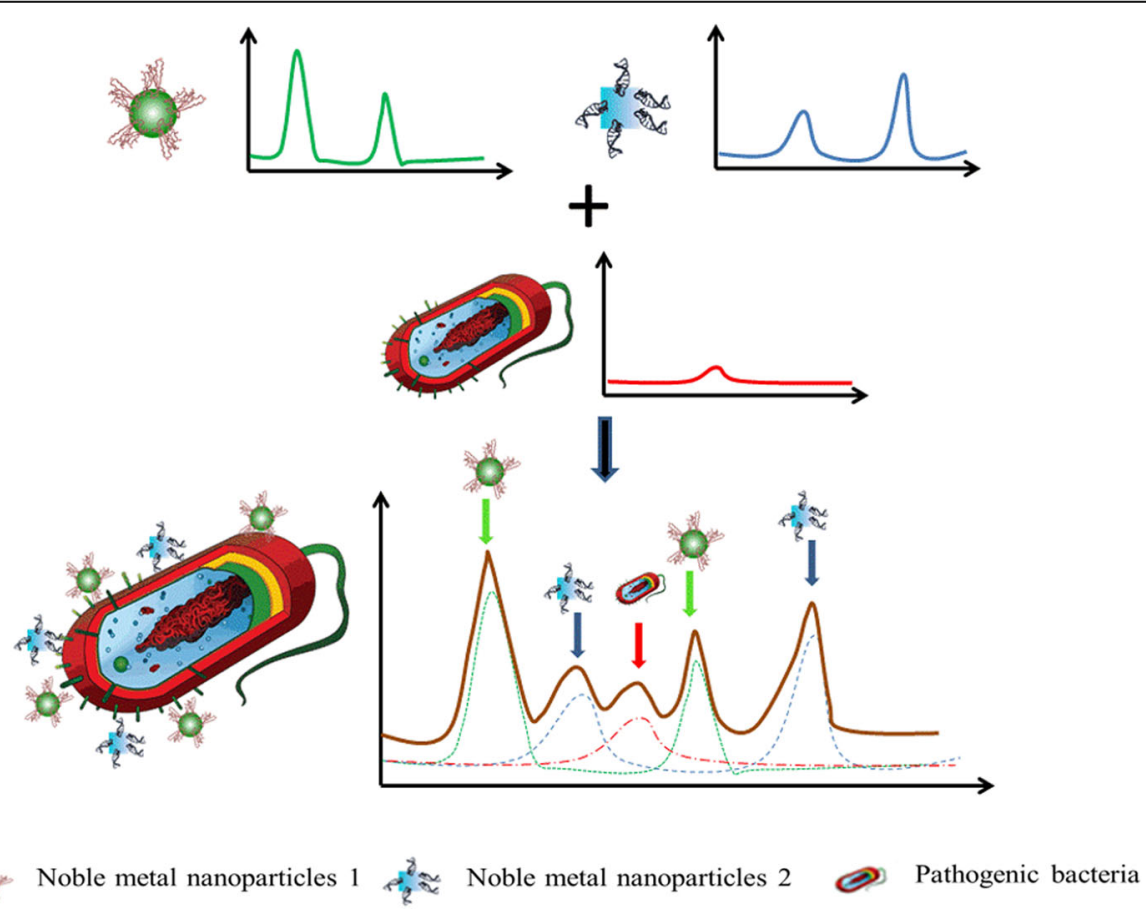

Fig. 2 Schematic of Multiplex self-referencing pathogen recognition using SERS molecular probes 


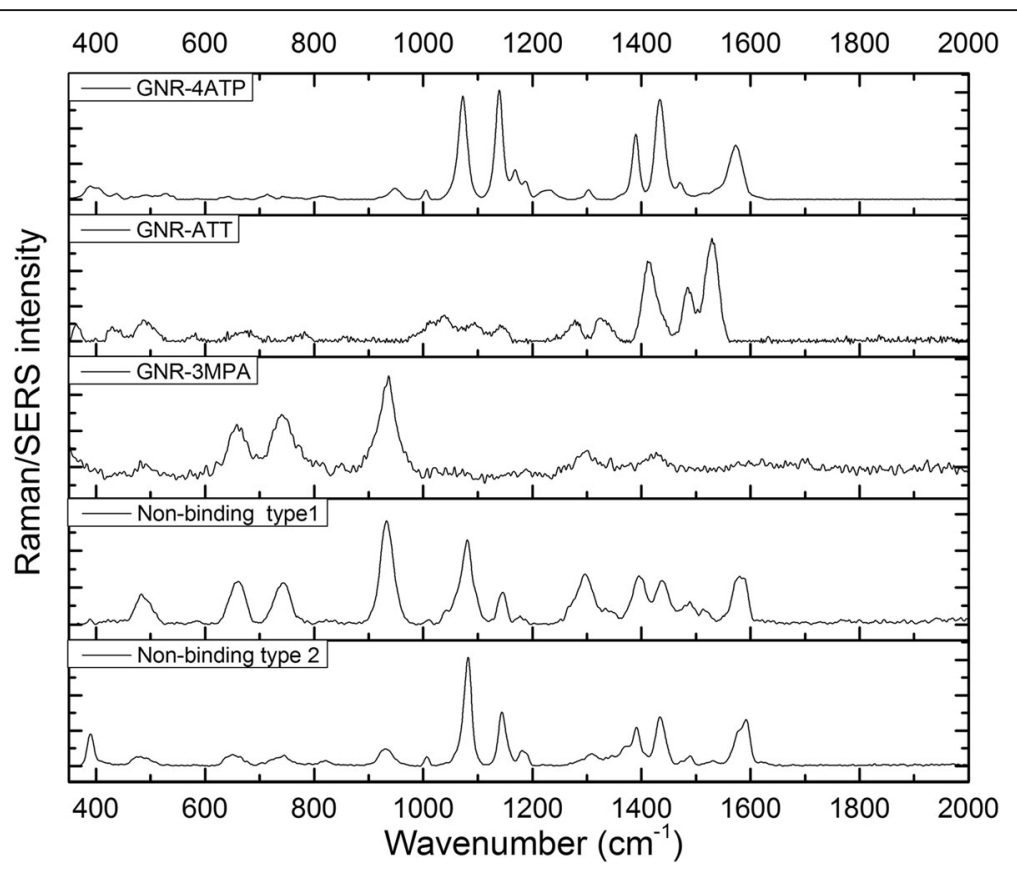

Fig. 3 SERS spectra of three nanoprobes. Upper three spectra: nanorod-4ATP-monoclonal antibody; nanorod-ATT-monoclonal antibody; nanocage-3MPA-polyclonal antibody. Down two spectra: SERS spectra of two subtypes of non-binding

In our self-referencing scheme, the SERS signatures of the target bacteria were observed superimposed with the SERS signals of the Raman tags. The assessment through the dual signals (superimposed target and tag Raman signatures) supported a specific recognition of the targets in a single step with no washing/separation needed. However, the complex nature of the SERS imparts the implementation of the self-referencing scheme with a lot of variations. Dual signals could only be observed when the conjugated bacterial cell wall components fall into the hotspot regions of the nanoprobes. Hot spots are highly localized regions of intense local field enhancement believed to be caused by local surface plasmon resonances. In practice, the variation of nanoprobe conjugation location and density on the surface of the bacterial cells was very high. Therefore, the enhanced-type spectra were not expected in every single measurement. Among all the spectra collected, most were non-enhanced Raman spectra (data not shown) in which the significantly enhanced Raman fingerprint signatures were not identifiable from both Raman tag and bacteria; and 20\% of the acquired spectra were considered as SERS spectra. Among the SERS spectra, two types could be identified: one was nonbinding (probe alone) type in which only the featured peaks from three Raman tag molecules could be found, indicating that the probes were not bound to bacterial cells; the other was binding (dual signal) type in which both Raman tags' peaks and bacterial peaks were significantly enhanced. The multiplex scheme we employed to detect multiple epitopes further added to the complication of the analysis. As shown in Fig. 3, even the non-binding spectra do not always show identical characteristics: in some measurements the three probe signals were not all detectable at the same intensity level. The randomly occurrence of hot spots is to blame for this inconsistency.

The average probe (i.e., non-binding) and dual (i.e., binding) types SERS spectra are shown in Fig. 4a. The peaks from Raman tags are assigned at the top of this figure. Besides the Raman tags SERS peaks, some weaker but identifiable peaks could be found in the microbial information rich region (500-1000 $\left.\mathrm{cm}^{-1}\right)$. The peak assignment for bacterial components (bacteria at $10^{1} \mathrm{CFU} / \mathrm{mL}$ ) in dual SERS spectra are shown in Fig. 4b. The pure and high-concentration E.coli $0157: H 7$ is also exhibited in this figure as reference (see Table 1 for peak assignment). Although the dual spectra of the sample can be easily differentiated from the probe-alone spectra of the control, the reproducibility of the SERS-based self-referencing analysis was still not ideal. The variations could be due to the nature of the SERS process, where even the same analyte (i.e., chemically heterogeneous bacterium) cannot be expected to repeatedly satisfy all of the criteria for SERS, namely, orientation, and presence within the range of the enhanced local optical field [34]. Furthermore, the surface enhancement of nanostructures is highly distance dependent and the electromagnetic field decays exponentially away from the nanoparticle's surface. Hence, the Raman tag molecules, as the nearest conjugated layer, exhibit the highest Raman peaks intensity. On contrast, the outside layer bacterial cell wall components show weaker peak intensity. Additionally, 

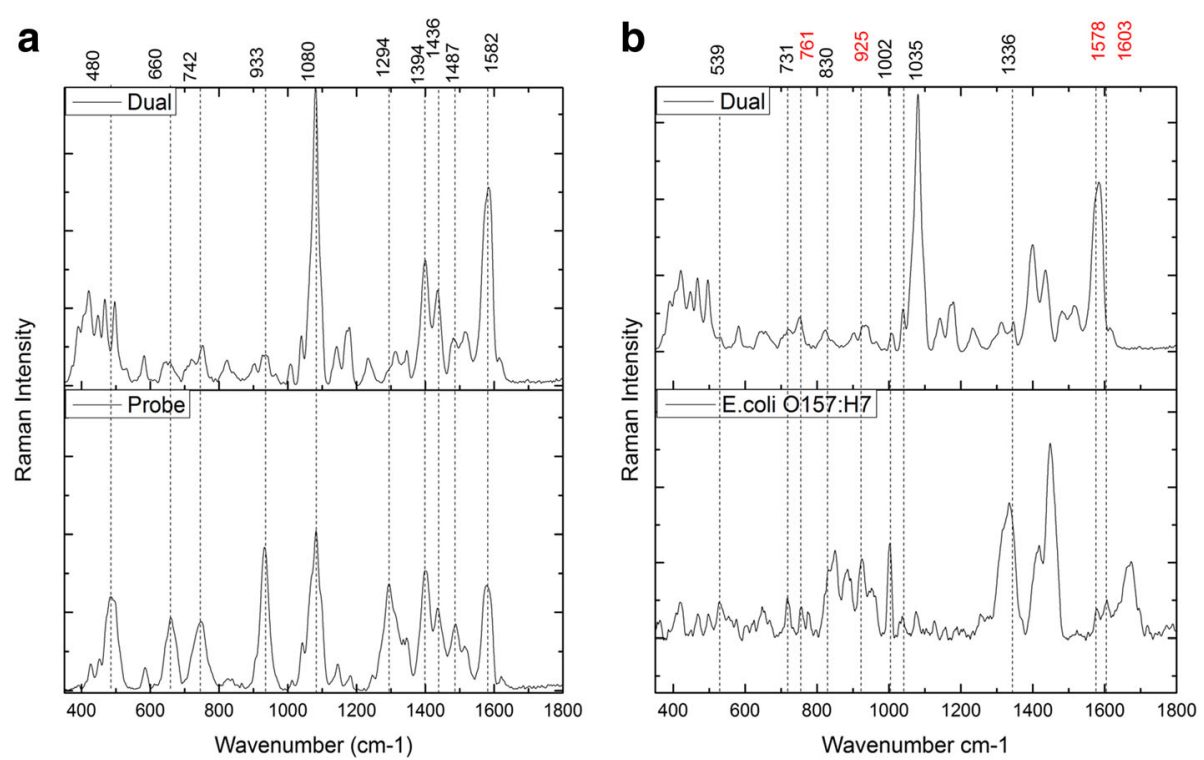

Fig. 4 SERS spectral results of bacterial samples using Nano-DEP microfluidic device. a SERS spectra and peak assignment of non-binding (probe signal) type and binding (dual signal) type (the marked peaks are the featured Raman peaks from three Raman tag molecules); (b) SERS spectra and peak assignment of binding type and normal Raman spectra of pure \& high-concentration E.coli O157: H7 bacteria (the marked peaks are the Raman signatures from target pathogen, the red color marked ones cannot be clearly identified in the dual-type spectra)

some of the important microbial components (marked as red color wavenumber) peaks could not be clearly identified due to the overlapping with the Raman tags featured signals. All these complexity makes the self-referencing scheme not very consistent. To assure consistent and reproducible detection is to be achieved, statistical analysis needs to be used.

\section{Multivariate statistical analysis for rapid discrimination and classification of target bacteria}

To qualify the spectral differences responsible for discrimination, the first 5 PC loadings (see Fig. 5) were

Table 1 Band assignment of E.coli 0157:H7 featured peaks shown in multiple self-referencing SERS measurement

\begin{tabular}{ll}
\hline Wavenumber $\left(\mathrm{cm}^{-1}\right)$ & Band assignment \\
\hline 1660 & Amide 1 vibration \\
1603 & Phenylalanine in protein \\
1578 & Adenine ring stretching \\
1336 & $\gamma\left(\mathrm{NH}_{2}\right)$ adenine, polyadenine, \\
& Phenylalanine in protein \\
1035 & Phenylalanine in protein \\
1002 & Phenylalanine in protein \\
925 & CN stretching \\
$830 / 850$ & Tyrosine \\
761 & Aromatic aminoacids/Tryptophan \\
731 & adenine, glycosidic ring mode, DNA \\
539 & S-S bond stretching \\
\hline
\end{tabular}

compared to find the most representative loading spectra that could be originated from bacterial targets. It is considered that the second PC loading spectrum showed the highest correlation with the bacterial Raman spectra. The spectral contribution from the second PC loading is assigned in Fig. 6, in comparison to the Raman spectra of the high-concentration E.coli O157:H7. The $2^{\text {nd }} \mathrm{PC}$ loading shows Raman signature bands of target pathogen matching at $731,850,1002,1035,1093,1331,1603$, $1660 \mathrm{~cm}^{-1}$ (see Table 1 for reference). This result further confirms the underlining mechanism of the selfreferencing detection scheme: binding to pathogen targets lead to detectable SERS signatures that differentiate dual SERS spectra from probe-alone spectra without separation of target-bound from unbound.

To further confirm the differentiation between positive signal (i.e., Dual spectra) and negative control (i.e., probe-alone spectra), a binary-based classification algorithm based on Support Vector Machine (SVM) was used in this study. Rather than performing prediction analysis using all of the spectroscopic information in the dataset, we use only those spectroscopic components with the strongest estimated correlation with bacterial target. After dimension reduction with PCA, we need to decide which principle components are important. The first 58 PCs, represented $80 \%$ of the total variance in the data set, were used for the following SVMs calculation. Linear kernel was used in our SVM model. The two types of SERS spectra (non-binding, probe alone and binding, dual) were collected from three separated 


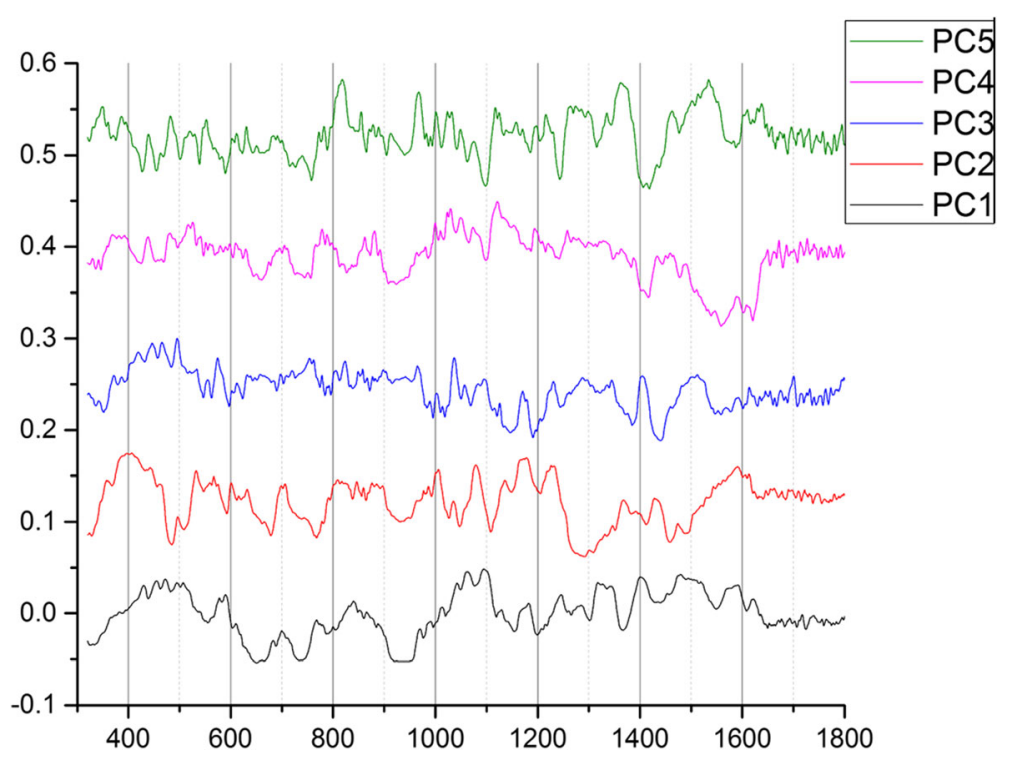

Fig. 5 The first 5 principal component loadings of the PCA performed on the SERS spectra acquired from multiplex antibodies functionalized Nanoprobes conjugating with E.coli O157: H7 bacteria sample

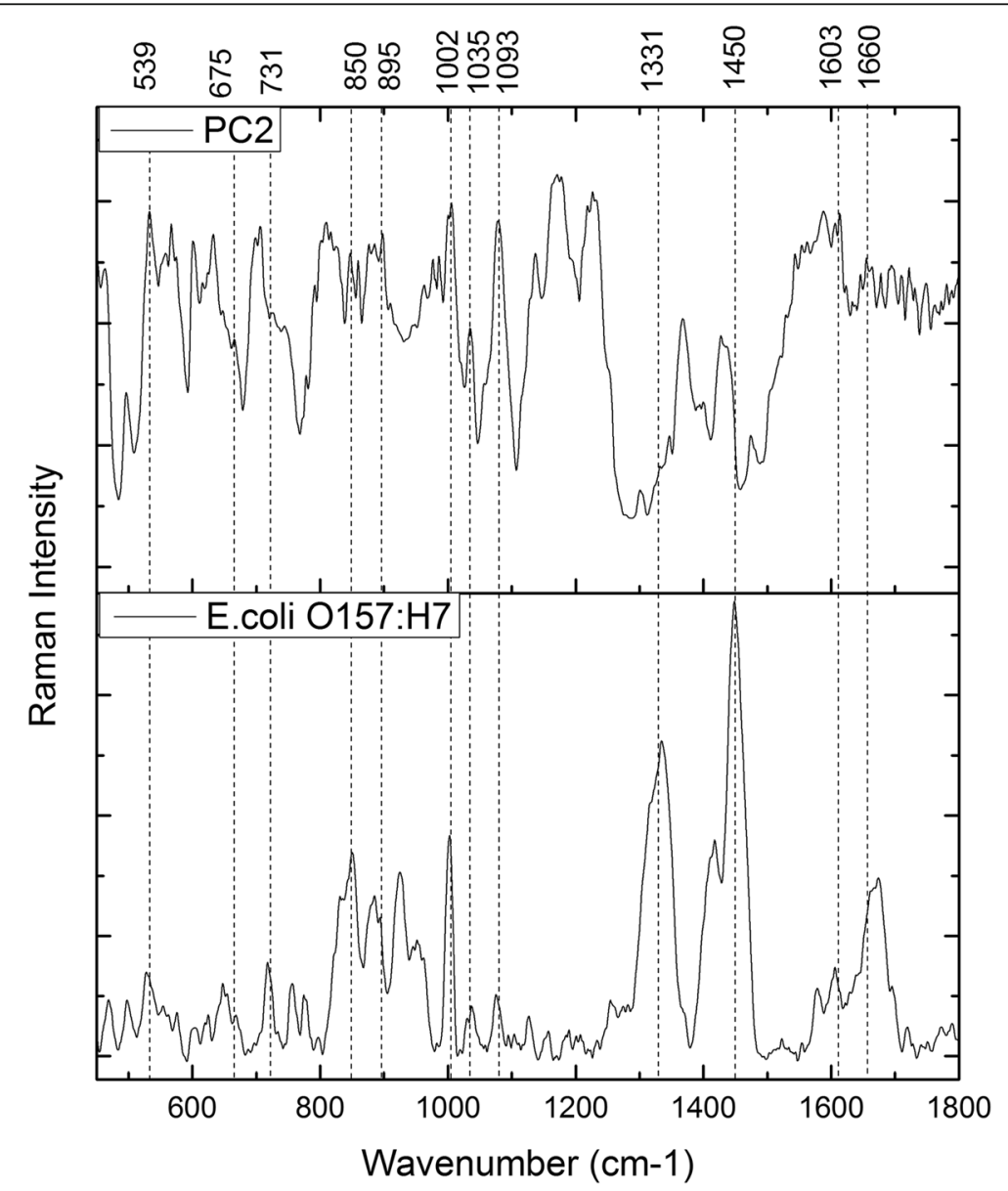

Fig. 6 The peak identification of PC2 spectral loading and the Raman spectrum of pure \& high-concentration Bacterial target cells 
batches of experiments. A sum of 166 spectra were used in the SVM testing, 79 were dual type; 87 were probe type SERS spectra. In this experiment the dataset is randomly split into two subsets: one containing 124 spectra was used to train the SVM model and the second dataset containing 42 spectra was used for the evaluation to validate the established SVM model by rotating the tested dataset into the same coordinate system as the training dataset. A shown in Fig. 7, the differentiation between the negative control and the positive I.D. is very good. The validation (Table 2) was evaluated by the accuracy percentage $(>95 \%)$. It should be noted that here the negatively control was E. coli $\mathrm{K} 12$ alone (at 100 $\mathrm{CFU} / \mathrm{mL}$ ), and the positive sample was a mixture of $E$. coli $\mathrm{K} 12$ and E. coli $\mathrm{O} 157: \mathrm{H7}$ (at 10:1 ratio), at $100 \mathrm{CFU} /$ $\mathrm{mL}$ (with $\mathrm{O} 157: \mathrm{H} 7$ at $10 \mathrm{CFU} / \mathrm{mL}$ ). All three nanoprobes were prepared to specifically bind to $E$. coli O157:H7 cells at three different epitopes. These results indicate that the self-referencing detection scheme allows the detection of a pathogen at very low level (10 $\mathrm{CFU} / \mathrm{mL}$ ) at the presence of 10 times higher non-target strains without any separation steps with very high fidelity, a specificity at sub-strain level.

\section{Enrichment of samples by using Nano-DEP microfluidic device}

Our results showed the LOD could be improved to $10^{1}$ $\mathrm{CFU} / \mathrm{mL}$ by using the multiple epitopes self-referencing recognition strategy. Even though this SERS-based scheme
Table 2 Result of the probe \& dual spectra validation with a SVM model (the first 58 PCs are used only)

\begin{tabular}{lll}
\hline & SVM testing & \\
\cline { 2 - 3 } prediction & Probe & Dual \\
\hline Probe & 22 & 0 \\
Dual & 1 & 19
\end{tabular}

could already provide such ultrasensitive and rapid detection results, the sensitivity needs further improvement for it to be a frontline solution to pathogen detection. It has been reported that the infectious dose of E.coli O157:H7 bacteria is only 10 cells per gram of food and $0.2 \mathrm{CFU} / \mathrm{mL}$ in environmental sample, which underlines the desirability for extremely sensitive and specific pathogen detection [35].

In this study, we integrated a Nano-DEP microfluidic device into our detection platform. The nano-DEP device was used to enrich the samples before mixing with nanoprobes. Dieletrophoresis (DEP), a nondestructive electrokinetic transport mechanism, has been used to concentrate and separate various types of cells, especially microorganisms. DEP is the movement of particles due to polarization effects in non-uniform electric fields [36]. Nano-DEP utilizes carbon nanotube electrodes that generate DEP force that is one magnitude larger than that of normal DEP devices, hence the trapping/capturing efficiency is significantly improved [37]. This is critical when the sample to be concentrated is already a much diluted sample (e.g., 1-10 $\mathrm{CFU} / \mathrm{mL}$ ). Figure 8 illustrates the Nano-DEP microfluidic

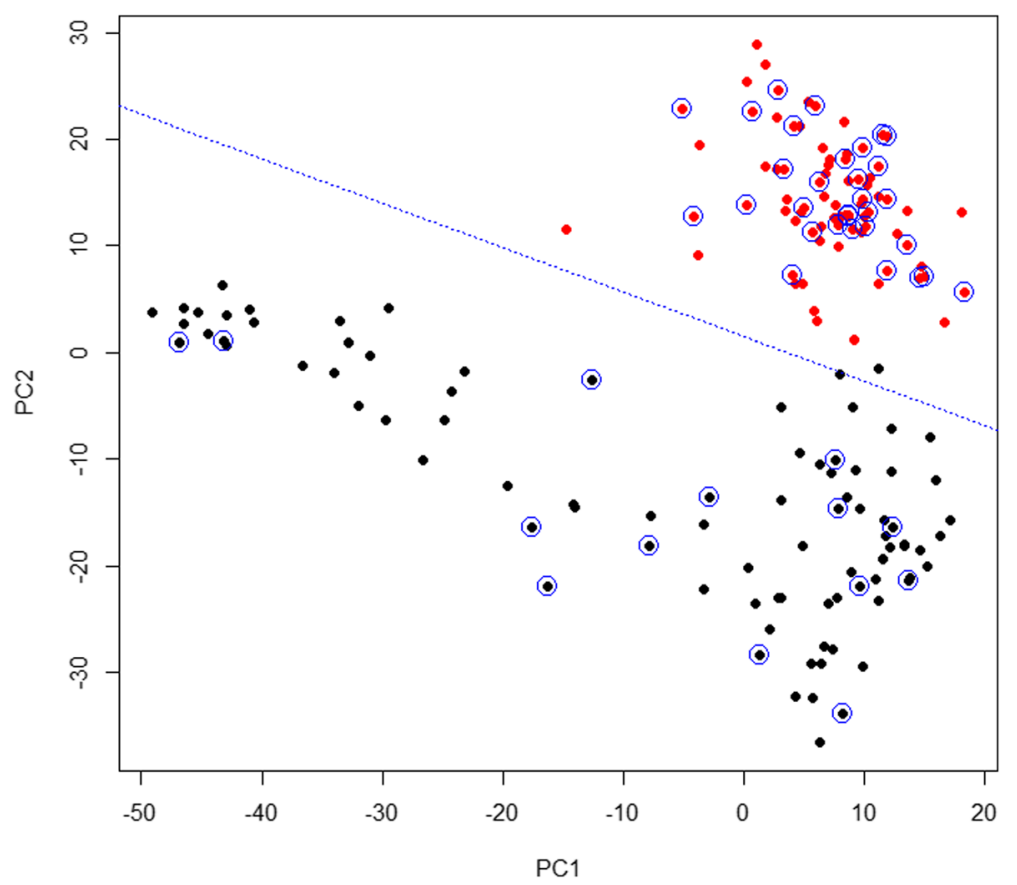

Fig. 7 Classification of binding type and non-binding type spectra using SVM. Red dots represent binding type spectra; black dots represent nonbinding type spectra. The dash line is the hyperplane showing the optimal linear separation. The blue circles indicate the support vectors 


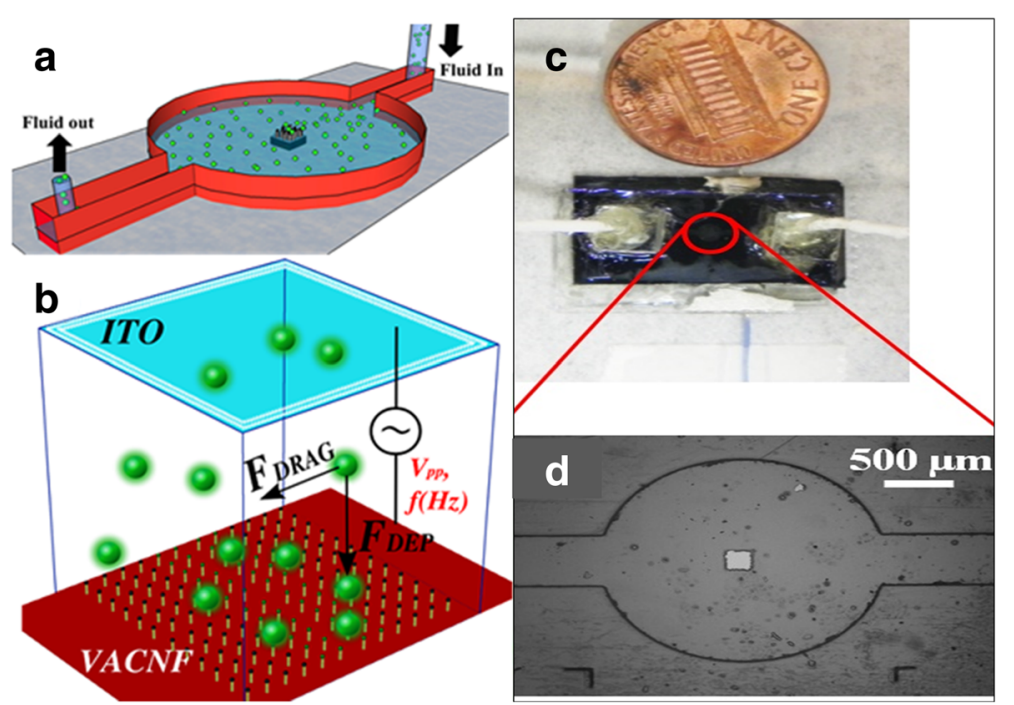

Fig. 8 Schematic illustration of Nano-DEP microfluidic device. a the design of reservoir and fluid in/out channel; (b) carbon nanofiber nanoelectrode arrays (CNFNEAs) were embedded into this microfluidic device; (c) the actual size of this microfluidic device; (d) the dimension of the reservoir under microscope

device used in this study for single cell trapping. In this labon-a-chip setup, the integrated vertical aligned carbon nanofiber nanoelectrode tip (in the square position, Fig. 8d) displayed an extremely high electric field gradient $\left(10^{20} \mathrm{~V}^{2}\right.$ $\mathrm{m}^{-3}$ ) when applied a voltage source, generating the socalled DEP force, a force exerted on a suspended dielectric particle (microbe) in the presence of a non-uniform electric field. The nano-DEP is strong enough to achieve cells trapping at high throughput by overcoming the hydrodynamic drag influence (Fig. 8b). The Nano-DEP device is considered act as a concentrator of target pathogen cells in this study. By passing through the bacteria-containing water samples $\left(10^{\circ} \mathrm{CFU} / \mathrm{mL}\right.$ or below) into the device for certain time periods when voltage turning on, the number of the cells trapped on the electrodes increase gradually. After certain duration of enrichment, the trapped target cells are released in one burst when the voltage is turned off, and they can be collected to a higher and detectable concentration level $\left(10^{1} \mathrm{CFU} / \mathrm{mL}\right)$.

The concentrated samples (volume from $1 \mathrm{~mL}$ to100 $\mu \mathrm{L}$; concentration from $10^{0}$ to $10^{1} \mathrm{CFU} / \mathrm{mL}$ ) were collected by using microfluidic device. Two different mixed cell suspensions with different concentration ratios (between E.coli O157:H7 and E.coli K12, namely O:K) were chosen in the testing. One was $1 \mathrm{CFU} / \mathrm{mL}$ (E.coli O157: H7): $1 \mathrm{CFU} / \mathrm{mL}$ (E.coli K12); the other was $1 \mathrm{CFU} / \mathrm{mL}$ (E.coli O157:H7): $10 \mathrm{CFU} / \mathrm{mL}$ (E.coli K 12). The concentration of original mixed cell suspension and the concentrated suspension were validated by plate counting. The results are shown in Fig. 9. From the results of both

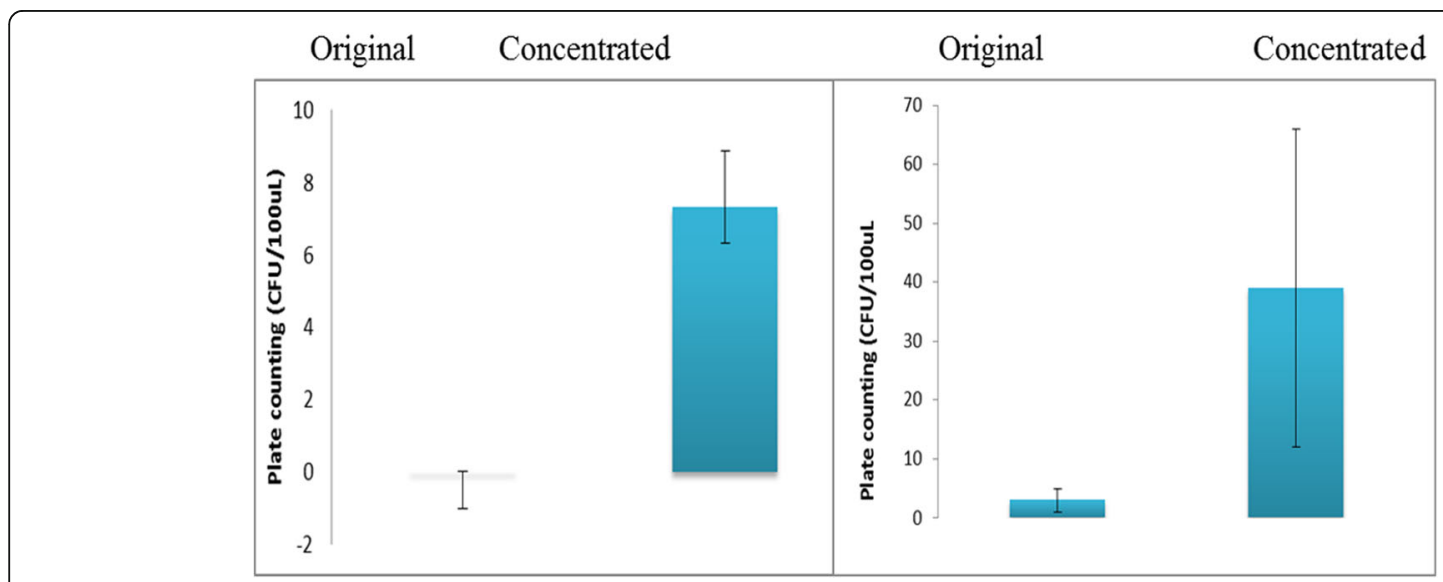

Fig. 9 Plate counting results for two concentration ratios. Left: O:K=1:1 (CFU/mL in original solution); right: O:K=1:10 (CFU/mL in original solution) 
mixture tests, the 10 times enrichment efficiency was demonstrated. Since the multiplex self-referencing SERS nanoprobes can provide a high sensitive detection of the target bacteria, it is satisfied to obtain 10 times concentrating in the pre-enrich step using Nano-DEP.

\section{Conclusion}

This novel multiplex self-referencing SERS pathogen detection scheme offered high sensitivity $\left(10^{1} \mathrm{CFU} / \mathrm{mL}\right)$ and strain level discrimination by measuring the superimposed SERS signatures with multiple characteristic peaks. Furthermore, the superimposed spectra could be obtained directly with no washing being performed. Compared to the ELISA kits, this platform successfully isolated and identified bacteria in water samples without the need for repeated wash steps and secondary antibody reporting, hence significantly reducing the operation processes, detection time and the cost. In addition, this platform integrated with an excellent separation and concentration apparatus of Nano-DEP microfluidic device further improves the limit of detection (LOD) to $10^{\circ} \mathrm{CFU} / \mathrm{mL}$. The integration of microfluidic devices with SERS detection yielded simple and miniaturized instrumentation that was suitable for the detection and characterization of small volume of chemical and biological analytes with high sensitivity and specificity. Multivariate statistical analysis techniques (PCA and SVM) firmly confirms the positive identification of targets in the presence of overwhelming non-target interference, with a detection accuracy above $95 \%$. It has the potential to become a powerful, highly sensitive biosensor for onsite detection of pathogens at extremely low levels.

\section{Acknowledgements}

Not applicable.

\section{Funding}

Research reported in this publication was supported by lowa State University Foundation and lowa VA medical center.

\section{Availability of data and material}

Data available in a public (institutional, general or subject specific) repository that issues datasets with DOls (non-mandated deposition).

\section{Authors' contributions}

CW carried out the nanoparticles/probes fabrication, characterization, collected SERS spectra data, performed the statistical data analysis, and drafted the manuscript. FM fabricated and tested the Nano-DEP microfluidic device. Dr. CY and Dr. JL participated in its design and helped to revise the manuscript. All authors read and approved the final manuscript.

\section{Competing interests}

The authors declare that they have no competing interests.

\section{Consent for publication}

Not applicable.

Ethics approval and consent to participate Not applicable.

\section{Author details}

${ }^{1}$ Agricultural and Biosystems Engineering Department, lowa State University, Ames, IA 50011, USA. ²Chemistry department, Kansas State University, Manhattan, KS 66506, USA.

Received: 20 October 2016 Accepted: 2 February 2017

Published online: 14 February 2017

\section{References}

1. Leonard P, Hearty S, Brennan J, Dunne L, Quinn J, Chakraborty T, O'Kennedy R. Advances in biosensors for detection of pathogens in food and water. Enzyme Microb Technol. 2003;32(1):3-13.

2. Lazcka O, Campo FJD, Muñoz FX. Pathogen detection: A perspective of traditional methods and biosensors. Biosens Bioelectron. 2007;22(7):1205-17.

3. Lam H, Kostov Y. Optical Instrumentation for Bioprocess Monitoring. Optical Sensor Systems in Biotechnology. Advances in Biochemical Engineering/ Biotechnology Series 116. New York: Springer Berlin Heidelberg; 2009: p. 125-42.

4. Ahmed A, Rushworth JV, Hirst NA, Millner PA. Biosensors for Whole-Cell Bacterial Detection. Clin Microbiol Rev. 2014;27(3):631-46.

5. Torun Ö, Hakkı Boyacı I, Temür E, Tamer U. Comparison of sensing strategies in SPR biosensor for rapid and sensitive enumeration of bacteria. Biosens Bioelectron. 2012;37(1):53-60.

6. Arya SK, Singh A, Naidoo R, Wu P, McDermott MT, Evoy S. Chemically immobilized T4-bacteriophage for specific Escherichia coli detection using surface plasmon resonance. Analyst. 2011;136(3):486-92.

7. Straub TM, Dockendorff BP, Quiñonez-Díaz MD, Valdez CO, Shutthanandan J, Tarasevich BJ, Grate JW, Bruckner-Lea CJ. Automated methods for multiplexed pathogen detection. J Microbiol Methods. 2005;62(3):303-16.

8. Kim JH, Mun S, Ko HU, Yun GY, Kim J. Disposable chemical sensors and biosensors made on cellulose paper. Nanotechnology. 2014;25(9):092001.

9. Wu X, Xu C, Tripp RA, Huang Y-w, Zhao Y. Detection and differentiation of foodborne pathogenic bacteria in mung bean sprouts using field deployable label-free SERS devices. Analyst. 2013;138(10):3005-12.

10. Siti M, Wahyudiono, Noriharu T, Hideki K, Koichi S, Motonobu G. Fabrication of gold and silver nanoparticles with pulsed laser ablation under pressurized $\mathrm{CO}_{2}$. Adv Nat Sci Nanosci Nanotechnol. 2013;4(4):045011.

11. Driscoll AJ, Harpster MH, Johnson PA. The development of surfaceenhanced Raman scattering as a detection modality for portable in vitro diagnostics: progress and challenges. Phys Chem Chem Phys. 2013;15(47): 20415-33.

12. Stadler J, Schmid T, Zenobi R. Nanoscale Chemical Imaging Using TopIllumination Tip-Enhanced Raman Spectroscopy. Nano Lett. 2010;10(11): 4514-20.

13. Monica P, Monica B, Cosmin F, Simion A. Chitosan-coated anisotropic silver nanoparticles as a SERS substrate for single-molecule detection. Nanotechnology. 2012;23(5):055501.

14. Yang D, Zhou H, Haisch C, Niessner R, Ying Y. Reproducible E. coli detection based on label-free SERS and mapping. Talanta. 2016;146:457-63.

15. Zhou H, Yang D, Ivleva NP, Mircescu NE, Niessner R, Haisch C. SERS Detection of Bacteria in Water by in Situ Coating with Ag Nanoparticles. Anal Chem. 2014:86(3):1525-33.

16. Kniggendorf A-K, Gaul TW, Meinhardt-Wollweber M. Hierarchical Cluster Analysis (HCA) of Microorganisms: An Assessment of Algorithms for Resonance Raman Spectra. Appl Spectrosc. 2011;65(2):165-73.

17. Yang X, Zhang AY, Wheeler DA, Bond TC, Gu C, Li Y. Direct moleculespecific glucose detection by Raman spectroscopy based on photonic crystal fiber. Anal Bioanal Chem. 2012;402(2):687-91.

18. Walter A, Marz A, Schumacher W, Rosch P, Popp J. Towards a fast, high specific and reliable discrimination of bacteria on strain level by means of SERS in a microfluidic device. Lab Chip. 2011;11(6):1013-21.

19. Su L, Zhang P, Zheng D, Wang Y-j-q, Zhong R-g. Rapid detection of Escherichia coli and Salmonella typhimurium by surface-enhanced Raman scattering. Optoelectron Lett. 2015;11(2):157-60.

20. Stöckel S, Kirchhoff J, Neugebauer U, Rösch P, Popp J. The application of Raman spectroscopy for the detection and identification of microorganisms. J Raman Spectrosc. 2016:47(1):89-109.

21. Páez-Avilés C, Juanola-Feliu E, Punter-Villagrasa J, del Moral Zamora B, Homs-Corbera A, Colomer-Farrarons J, Miribel-Català PL, Samitier J. Combined Dielectrophoresis and Impedance Systems for Bacteria 
Analysis in Microfluidic On-Chip Platforms. Sensors (Basel, Switzerland). 2016;16(9):1514.

22. Khoshmanesh K, Baratchi S, Tovar-Lopez FJ, Nahavandi S, Wlodkowic D, Mitchell A, Kalantar-zadeh K. On-chip separation of Lactobacillus bacteria from yeasts using dielectrophoresis. Microfluid Nanofluid. 2012;12(1):597-606.

23. Khoshmanesh K, Nahavandi S, Baratchi S, Mitchell A, Kalantar-zadeh K. Dielectrophoretic platforms for bio-microfluidic systems. Microfluid Nanofluid. 2011;26(5):1800-14.

24. Yoon-Kyoung C, Tae-hyeong K, Jeong-Gun L. On-chip concentration of bacteria using a 3D dielectrophoretic chip and subsequent laser-based DNA extraction in the same chip. J Micromech Microeng. 2010;20(6):065010.

25. Lin H-Y, Huang C-H, Hsieh W-H, Liu L-H, Lin Y-C, Chu C-C, Wang S-T, Kuo IT, Chau L-K, Yang C-Y. On-line SERS Detection of Single Bacterium Using Novel SERS Nanoprobes and A Microfluidic Dielectrophoresis Device. Small. 2014;10(22):4700-10

26. Cheng IF, Lin C-C, Lin D-Y, Chang H-C. A dielectrophoretic chip with a roughened metal surface for on-chip surface-enhanced Raman scattering analysis of bacteria. Biomicrofluidics. 2010;4(3):034104.

27. Cherukulappurath S, Lee SH, Campos A, Haynes CL, Oh S-H. Rapid and Sensitive in Situ SERS Detection Using Dielectrophoresis. Chem Mater. 2014; 26(7):2445-52

28. Cheng IF, Chang H-C, Chen T-Y, Hu C, Yang F-L. Rapid ( $<5$ min) Identification of Pathogen in Human Blood by Electrokinetic Concentration and Surface-Enhanced Raman Spectroscopy. Sci Rep. 2013;3:2365.

29. Nikoobakht B, El-Sayed MA. Preparation and Growth Mechanism of Gold Nanorods (NRs) Using Seed-Mediated Growth Method. Chem Mater. 2003; 15(10):1957-62.

30. Skrabalak SE, Au L, Li X, Xia Y. Facile synthesis of Ag nanocubes and Au nanocages. Nat Protocols. 2007;2(9):2182-90.

31. Zhang Z-M, Chen S, Liang Y-Z, Liu Z-X, Zhang Q-M, Ding L-X, Ye F, Zhou H. An intelligent background-correction algorithm for highly fluorescent samples in Raman spectroscopy. J Raman Spectrosc. 2010:41(6):659-69.

32. Xiao N, Wang C, Yu C. A Self-Referencing Detection of Microorganisms Using Surface Enhanced Raman Scattering Nanoprobes in a Test-in-a-Tube Platform. Biosensors. 2013;3(3):312-26.

33. Harper MM, McKeating KS, Faulds K. Recent developments and future directions in SERS for bioanalysis. Phys Chem Chem Phys. 2013;15(15):5312-28.

34. Jarvis RM, Goodacre R. Discrimination of Bacteria Using Surface-Enhanced Raman Spectroscopy. Anal Chem. 2004;76(1):40-7.

35. Johnson L, Rose J, Sharar BE, Ransom AK, Lattuada GM, McNamara CP, A M. Methods Used for Detection and Recovery of Escherichia coli 0157:H7 Associated with a Food-Borne Disease Outbreak. J Food Prot. 1995;58(6):597-603.

36. Moncada-Hernández H, Lapizco-Encinas BH. Simultaneous concentration and separation of microorganisms: insulator-based dielectrophoretic approach. Anal Bioanal Chem. 2010;396(5):1805-16

37. Madiyar FR, Syed LU, Arumugam P, Li J. Electrical Capture and Detection of Microbes Using Dielectrophoresis at Nanoelectrode Arrays. Adv Appl Nanotechnol Agric. 2013;1143:109-24.

\section{Submit your next manuscript to BioMed Central and we will help you at every step:}

- We accept pre-submission inquiries

- Our selector tool helps you to find the most relevant journal

- We provide round the clock customer support

- Convenient online submission

- Thorough peer review

- Inclusion in PubMed and all major indexing services

- Maximum visibility for your research

Submit your manuscript at www.biomedcentral.com/submit

) Biomed Central 